\title{
Transparency Maximization Methodology for Haptic Devices
}

\author{
Kostas Vlachos and Evangelos Papadopoulos, Senior Member, IEEE
}

\begin{abstract}
In this paper, a design methodology is presented aimed at maximizing haptic device transparency, as seen from the user side. The methodology developed focuses on endpoint side fidelity, and optimizes not only mechanism dimensions, but also all relevant design parameters including relative position of endpoint desired path to device location, motor transmission ratios, and rotor inertias or motor sizes. The methodology is applied to a 5-degree-of-freedom (5-DOF) haptic device, part of a training medical urological simulator, and is applicable to any haptic mechanism. The transparency maximization is achieved using a multivariable optimization approach and an objective function including mechanism-induced parasitic torques/forces and motor and transmission parameters, as seen from the user side, under several constraints. The objective function and the kinematical and operational constraints are described and discussed. A new 5-DOF haptic mechanism is constructed according to the developed procedure, resulting in a substantially improved device with respect to an existing one, developed with a standard optimization method.
\end{abstract}

Index Terms-Haptic devices, multivariable optimization, training medical simulators.

\section{INTRODUCTION}

$\mathbf{T}$ HE quality of a haptic device is often judged by its transparency characteristics. Transparency is associated with the absence of haptic device-induced parasitic torques/forces during its motion, e.g., mass/inertia, gravity forces, and friction. Transparency is even more important for haptic devices used in simulators, where reality must be simulated reliably. Simulators are now an accepted tool in the training of surgeons because of the advantages they offer [1]. One can distinguish two trends in the development of medical simulators. The first is characterized by the use of general-purpose haptic devices, like the Phantom or the Freedom-7 [2], [3]. The second trend is characterized by the use of devices designed for a specific operation [4]-[6].

Optimization techniques have been used in improving the performance of mechanisms and manipulators. The inertial and acceleration characteristics of manipulators have been discussed in [7]. Optimization techniques were used to determine the minimum inertial properties and the maximum achievable acceleration of the end-effector in every direction over the workspace. Using an extensive search procedure, alternative mechanism designs were evaluated to result in a 4-degree-of-freedom (4-DOF) haptic mechanism optimum design that requires minimum continuous stall motor torques [8]. A global isotropy index has been

Manuscript received January 24, 2005; revised July 29, 2005. Recommended by Guest Editor Prof. Chen. This work was cofunded by the European Social Fund (75\%) and National Resources (25\%)-(EPEAEK II)-PYTHAGORAS.

The authors are with the Department of Mechanical Engineering, National Technical University of Athens, 15780 Athens, Greece (e-mail: kostaswl@central.ntua.gr; egpapado@central.ntua.gr).

Digital Object Identifier 10.1109/TMECH.2006.875561 proposed to quantify a configuration-independent isotropy of a robot's Jacobian or mass matrix [9]. This index was used to compare the performance of three manipulators, including two parallel and one hybrid robot [10]. A 2-degree-of-freedom (2-DOF) haptic device was optimized with respect to workspace, intrusion, inertia, response, and structural properties [11]. The architecture of a parallel redundant mechanism has been optimized from a kinematical viewpoint [12]. The dexterity, uniformity, and actuator forces have been investigated as potential objective functions. The joint redundancy is proposed for minimization of the device dynamics-induced parasitic torques/forces and friction [13]. Vlachos et al. have presented the design of a 5-DOF haptic interface, designed to exhibit a small condition number and mass, and satisfy several kinematical constraints [6]. The device was optimized using a classical approach and a multiobjective optimization technique [14].

This paper presents a multivariable optimization methodology resulting in haptic devices with maximum transparency. Since haptic devices interface with a user at their endpoint (user side), the goal of the optimization methodology is to establish a maximum transparency as seen from the user side. The methodology is exemplified using a 5-DOF haptic device, part of a training medical urological simulator, and is applicable to any haptic mechanism. The objective function employed in the minimization process includes the mass and inertia elements of the mechanism, and the motor and transmission characteristics of the device, as seen from the user side. The optimal design is achieved for a family of possible operational paths around a nominal one. The methodology results in an optimum: 1) mechanism geometry; 2) location of the path with respect to the haptic device base; 3) balancing weights; 4) balancing weights locations; 5) motor transmission ratio; and 6) main motor selection. The proposed optimization methodology is suitable for any actuated mechanism that must be optimized along a given path. Optimization results, comparisons to past designs, and the haptic device that resulted are presented.

\section{INITIAL DESIGN FOR A HAPTIC DEVICE}

As part of our previous work on the development of a training medical urological simulator, a haptic mechanism was designed that consisted of a five-bar parallel linkage with two translational DOF in series to a spherical joint with three rotational DOF [6]. The basic device configuration and the nominal endpoint path are shown in Fig. 1.

Initial optimization results based on standard approaches are presented briefly and used later as benchmarks for the methodology described in this paper. 


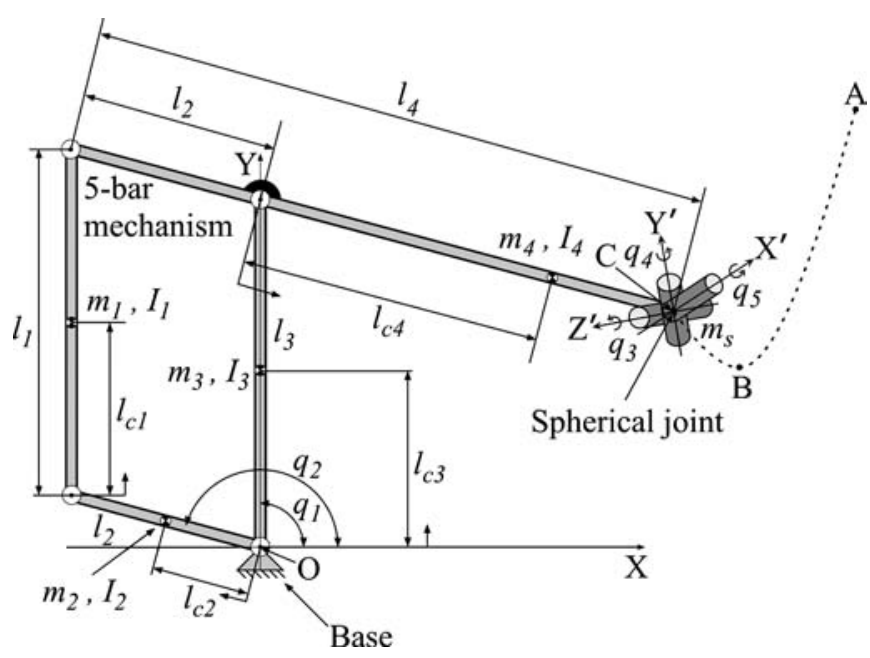

Fig. 1. Basic device configuration and the nominal endpoint path.

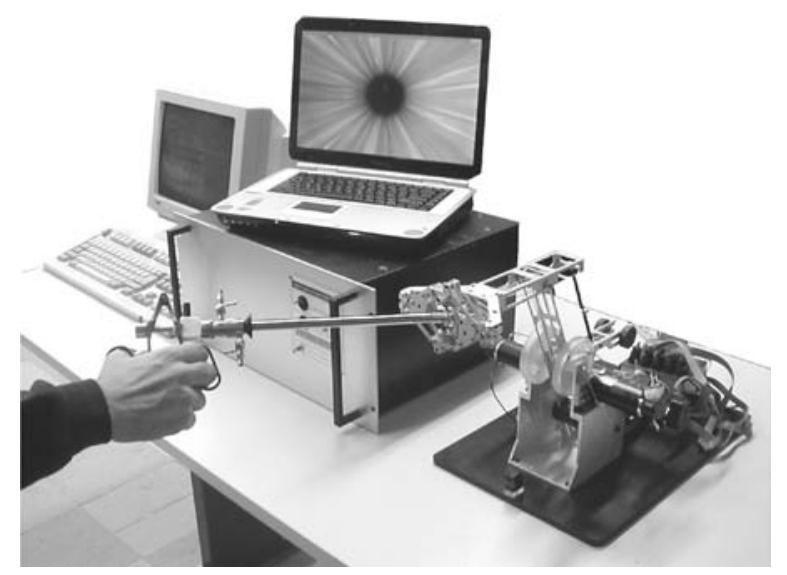

Fig. 2. First prototype of the haptic device based on the IOD as part of the training urological simulator.

\section{A. Initial Optimization and Design (IOD)}

To reduce mechanism moving mass and inertia, it was decided that all actuators should be placed at the base. This decision lead to the need for a transmission system and a design based on capstans, and tendon drives was selected.

In the first version of the haptic device, the four link lengths $l_{1}, l_{2}, l_{3}$, and $l_{4}$ of the five-bar mechanism, see Fig. 1, were optimized in order to minimize the condition number of the mechanism, under kinematical and structural constraints. In this paper, the condition number of a mechanism is defined as the ratio of the largest to the smallest singular value of the product $\mathbf{J}^{T} \mathbf{J}$, where $\mathbf{J}$ is the Jacobian matrix that relates endpoint Cartesian and angular velocities to motor rates. The nominal path $\mathrm{ABC}$ in Fig. 1 was fixed in space relative to the mechanism base in an ad hoc manner. The optimization was implemented in the joint space [6]. The haptic device that resulted from the IOD procedure is shown in Fig. 2, together with other elements of the developed training simulator.

\section{B. Joint Space Optimization (JSO)}

An attempt for a more comprehensive optimization was presented in [14], where a multiobjective optimization methodology was used. The optimization goal was to find the mechanism link lengths $l_{1}, l_{2}, l_{3}$, and $l_{4}$, and the location of the path $\mathrm{ABC}$ with respect to the basepoint $O$, see Fig. 1, so that mass, inertia, and friction at the joints of the five-bar mechanism are minimized. Similar to the IOD, this optimization dealt with the linkage and was defined in the joint space employing a classical mechanism approach.

Two objective functions were defined, the first focusing at mass/inertia optimization and the second at joint friction. The first function was chosen to be the sum of the link lengths

$$
f_{1}=l_{1}+l_{2}+l_{3}+l_{4-2} .
$$

Mass and inertia are functions of link lengths; therefore, minimization of this sum implicitly results in the minimization of mechanism mass and inertia.

The second objective function was the sum of the infinity norms of vectors $\boldsymbol{T}_{1}$ and $\boldsymbol{T}_{2}$

$$
f_{2}=w_{1} \operatorname{Norm}\left(\boldsymbol{T}_{1}, \text { inf }\right)+w_{2} \operatorname{Norm}\left(\boldsymbol{T}_{2}, \text { inf }\right) .
$$

Vectors $\boldsymbol{T}_{1}$ and $\boldsymbol{T}_{2}$ have as elements the actuator torques along $k$ segments of the typical path. The methodology results in an optimum mechanism geometry, as defined by lengths $l_{1}, l_{2}, l_{3}$, and $l_{4}$, and location of the endoscope path endpoint, as defined by the location of point $C$ in Fig. $1, C_{x}, C_{y}$, with respect to the haptic device base.

\section{USER-SIDE OPTIMIZATION DESIGN}

Haptic devices, opposite to classical robotic mechanisms, exert forces and torques to the user through their end-effector. The quality of such devices depends on the fidelity of the forces/torques applied on the user. Optimization procedures that use various criteria or terms defined in a device's joint space, such as the initial optimization efforts described earlier, are useful, but do not yield the best possible results.

To achieve the best possible performance, minimization of the mechanism and actuation system parasitic forces and moments as seen from the user side is required. To this end, a haptic mechanism must be designed with minimum inertial terms $\tilde{\mathbf{M}}$, nonlinear velocity terms $\tilde{\boldsymbol{V}}$, and gravity terms $\tilde{\boldsymbol{G}}$ as seen from the user side. We refer to this optimization procedure as a userside optimization design (USOD).

Next, the objective function $f$ is defined in a multivariable optimization approach.

\section{A. Objective Function}

In a medical training simulator, a tissue model computes tissue forces/torques $f(\boldsymbol{x}, \boldsymbol{v}, \dot{\boldsymbol{v}})$ that must be applied to the user. For device fidelity, the motor torques must be

$$
\boldsymbol{\tau}=\mathbf{J}^{T}(\tilde{\mathbf{M}} \dot{\boldsymbol{v}}+\tilde{\boldsymbol{V}}+\tilde{\boldsymbol{G}}+f(\boldsymbol{x}, \boldsymbol{v}, \dot{\boldsymbol{v}}))
$$

where $\boldsymbol{x}$ corresponds to endpoint displacements, $\boldsymbol{v}$ to velocities, and $\dot{v}$ to accelerations. According to (3), the actuators 
will have to compensate for the mechanism-induced parasitic torques/forces in addition of applying the computed tissue forces/torques $f(\boldsymbol{x}, \boldsymbol{v}, \dot{\boldsymbol{v}})$ to the endoscope. This approach results in larger motors and inertias, is prone to modeling errors and complexity, and increases the computational load, decreasing drastically the available force feedback bandwidth. To avoid these problems, we simply require that the parasitic terms be ideally zero, i.e.,

$$
\tilde{\mathbf{M}} \dot{\boldsymbol{v}}+\tilde{\boldsymbol{V}}+\tilde{\boldsymbol{G}}=0 .
$$

Therefore, the objective function to be minimized is selected to be the vector norm of the sum of the parasitic terms

$$
f=\operatorname{Norm}(\tilde{\mathbf{M}} \boldsymbol{v}+\tilde{\boldsymbol{V}}+\tilde{\boldsymbol{G}}) .
$$

Note, however, that optimizing the mechanism at a given configuration point is not satisfactory, since the mechanism moves as its tip follows a path. In such a case, it is desirable to obtain an optimum design for the entire endpoint path $\mathrm{ABC}$ in Fig. 1. To this end, the path is divided in $k$ segments and the objective function is constructed as the sum of functions $f_{i}$, each defined at segment $k$

$$
f=\sum_{i=1}^{k} f_{i}=\sum_{i=1}^{k} w_{i} \operatorname{Norm}(\tilde{\mathbf{M}} \dot{\boldsymbol{v}}+\tilde{\boldsymbol{V}}+\tilde{\boldsymbol{G}})_{i}
$$

where $w_{i}$ are weights corresponding to the contribution of each segment. For example, if the entire path is equally important, then all $w_{i}=1$, whereas if some segments are more critical, then the corresponding $w_{i}$ will be larger than 1 . In the case of the haptic device presented here, the behavior of the mechanism is more critical at the insertion point $A$ in Fig. 1 and even more during the main urological operation phase, which occurs at point $C$ in Fig. 1. However, the mechanism must also exhibit good behavior along the insertion path $\mathrm{ABC}$, and therefore, path segments are taken into account in (6). The optimization becomes more accurate as the number of segments increases. Because the objective function along the path is smooth and to reduce the computational load, we chose $k=12$.

\section{B. Design Parameters}

The design parameters depend on mechanism structure and its use are embedded in the terms of the objective function $f$ (6). Here, the mechanism structure is based on a hybrid design that consists of a closed five-bar parallel linkage providing two translational DOF in series to a spherical joint with three rotational DOF. The device tip follows paths such as the one shown in Fig. 1.

The spherical joint was designed to be statically balanced and as small as possible, subject to kinematical and manufacturing constraints. It is taken into account as a point mass $m_{\mathrm{s}}$ located at the end of link 4, see Fig. 3, and its contribution is not optimized directly further. Note here that still, the proposed optimization method is applicable to any haptic device with any number of DOF.

To define the unknown design parameters, we make the following observations: First, the value of the objective function (6) depends on the size and the design of the mechanism, and

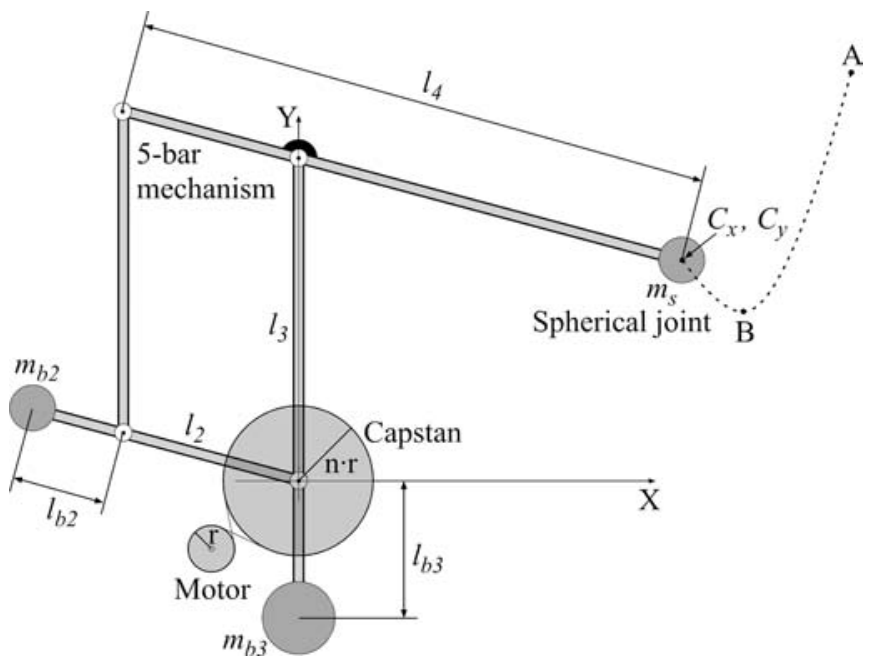

Fig. 3. Schematic view of the balanced 5-DOF haptic mechanism, of a random located typical path to follow, and the unknown design parameters.

the location and orientation of the typical path with respect to its base. The shape of the insertion path as well as the velocity and acceleration of the device tip along the path depend on operation practices, and therefore these are taken into consideration and do not affect the optimization. Next, (6) is examined in detail aiming at the selection of the specific design parameters.

Starting with the mass matrix $\tilde{\mathbf{M}}$, it is observed that its elements are functions of mechanism link lengths $l_{\mathrm{i}}$, masses $m_{\mathrm{i}}$, link moments of inertia $I_{\mathrm{i}}$, (i is a label for links), rotor moments of inertia $I_{m, \mathrm{j}}$, and transmission ratios $n_{\mathrm{j}}$, (j is a label for actuators). Link mass and inertia can be parameterized by link lengths. Therefore, $l_{2}, l_{3}=l_{1}$, and $l_{4}$ are selected as design parameters. The mass matrix $\tilde{\mathbf{M}}$ also contains rotor moments of inertia and transmission ratios. The motor capstan radii are selected as the smallest allowed by transmission design. The transmissions for the two five-bar DOF were taken equal to $n$ and this ratio is also included in the set of design parameters. Note here that by including the transmission ratio $n$ in the design parameters, we find not only the optimum ratio, but also the optimum motor. During the optimization procedure and for given maximum forces and torques at the endpoint, a change in $n$ results in a change in motors torques. Using a database of candidate motors, the optimization selects the motor with minimum rotor moment of inertia (included in $\tilde{\mathbf{M}}$ ), which can supply the required torque. The optimization results for the spherical joint transmission ratios were always equal to 1 . This is expected, because the maximum torques for these DOF are relative small, and small motors exist that produce the required torques without reduction and consequently with minimum apparent inertia. This is not the case with the parallel mechanism transmission, where the large required torques lead to combinations of motors with transmission reduction, out of which the procedure must select the best one.

The nonlinear velocity terms $\tilde{\boldsymbol{V}}$ are also functions of the above-mentioned parameters, therefore this term does not contribute additional design parameters.

The gravity terms $\tilde{G}$ are special in the sense that although they depend on the same parameters, they can be set to zero 
by proper design. One could exclude the gravity terms from (6) and try to completely eliminate them, by adding two balancing weights $m_{\mathrm{b} 2}$ and $m_{\mathrm{b} 3}$ on the extensions of links $2 l_{\mathrm{b} 2}$ and $3 l_{\mathrm{b} 3}$ in Fig. 3, [14]. This procedure would yield values for the products $m_{\mathrm{b} i} l_{\mathrm{b} i}(i=2,3)$ that would indeed eliminate $\tilde{\boldsymbol{G}}$. However, this does not necessarily minimize the other terms in (6). To see this, consider that a large balancing mass at a short distance may have the same effect as a small mass at a large distance from the basepoint, but with a different total system mass and inertia. Because of this reason, the gravity terms are not removed from (6), but instead four more design parameters are added, i.e., $m_{\mathrm{b} i}(i=2,3)$ and $l_{\mathrm{b} i}(i=2,3)$.

Next, the attention is focused on the relative location of the typical path with respect to the origin of the base frame of the device, since the overall size and configuration of the device depends on this. Indeed, for given link lengths, the device configuration as described by the joint angles $q_{j}$ depends on the location of the path relative to the device base. Because of the nature of the surgical operation, the path ABC always lies on the $X Y$-plane. Also, because the patient assumes a constant and predetermined position with respect to the vertical, the same applies to the orientation of path $A B C$. Therefore, the relative location of path $A B C$ with respect to the haptic interface base point $O$ is described by two parameters that locate one of its points with respect to $O$. We choose this point to be point $C$ with coordinates $C_{x}$ and $C_{y}$, and write expressions of the form

$$
q_{i}=q_{i}\left(C_{x}, C_{y}, l_{2}, l_{3}, l_{4}\right)
$$

Equation (7) introduces two more design parameters, i.e., $C_{x}, C_{y}$. The full set of design parameters is shown in Fig. 3 .

To complete the optimization procedure, one has to take into account several conflicting kinematical and implementation constraints. The constraints that were identified in this case are presented in Section III-C.

\section{Objective Function Constraints}

1) Inequality Constraints: An important constraint is that the mechanism tip must be in the device's workspace. The following inequality pair describes this constraint for all points along the path:

$$
\left(l_{3}-l_{4-2}\right) \leq \sqrt{x(s)^{2}+y(s)^{2}} \leq\left(l_{3}+l_{4-2}\right)
$$

where $l_{4-2} \equiv l_{4}-l_{2}$ and $x(s)$ and $y(s)$ are the coordinates of the mechanism tip along the path parameterized by its length $s$ and location $C_{x}, C_{y}$, see Fig. 3 .

In addition, the mechanism must be well conditioned at all configurations. It can be shown that the mechanism condition number is optimum when $l_{4-2}=l_{3}$ and $q_{2}-q_{1}=\pi / 2$, while it increases when $l_{4-2} \neq l_{1}$ and $q_{2}-q_{1} \neq \pi / 2$. The departure from the ideal isotropic conditions is described by

$$
\begin{gathered}
1-e_{1} \leq l_{3} / l_{4-2} \leq 1+e_{1} \\
(\pi / 2)-e_{2} \leq q_{2}-q_{1} \leq(\pi / 2)+e_{2}
\end{gathered}
$$

where $e_{1}$ and $e_{2}$ define the strictness of the above constraints.

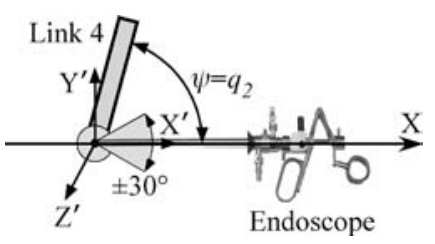

Fig. 4. Angle $\psi$ that is formed by link 4 and the endoscope at its initial orientation (parallel to $X$-axis) at point C.

It is important to note that (10) can be chosen so that it holds more strictly at specific points of the path followed by the mechanism tip. In this case, this would be the endpoint of the path, point $C$ in Fig. 3, where the main operation takes place. This introduces the following constraint:

$$
(\pi / 2)-e_{3} \leq q_{2, C}-q_{1, C} \leq(\pi / 2)+e_{3} .
$$

To make condition (11) stricter relative to (10), we choose $e_{2}>e_{3}$. The subscript $C$ in $q_{i, C}(i=1,2)$ denotes the value of angle $q_{i}$ at point $C$.

Another requirement results from implementing constraints such as collision avoidance. In this case, it is important to avoid collisions between link 4 and the endoscope. This can happen when the endoscope tip reaches point $C$ (main operation phase) and its orientation becomes parallel to the $X$-axis. At this point, the movements of the endoscope are mainly rotational, taking values $\pm 180^{\circ}$ around the $X^{\prime}$-axis, and $\pm 30^{\circ}$ around the $Z^{\prime}$ - and $Y^{\prime}$-axes. To avoid collisions between link 4 and the endoscope at $\mathrm{C}$, the angle $\psi$ formed between link 4 and the $X$-axis should allow the free rotation of the endoscope in an area much larger than the requirements, i.e., $\pm 70^{\circ}$, see Fig. 4. This requirement results in the following bounds for $q_{2}$ at point $C$ :

$$
70^{\circ} \leq q_{2, C} \leq 290^{\circ}
$$

which adds another optimization constraint.

During the training procedure, it is expected that the trainee will deviate from the nominal path. In this case, the device must have the capability not only to allow for these deviations, but also to maintain an optimum response. In collaboration with specialist surgeons, it was determined that the expected deviations are bounded by $e=0.01 \mathrm{~m}$ from the nominal path. This requirement leads to the following additional constraints:

$$
\begin{aligned}
& \left(l_{3}-l_{4-2}\right) \leq \sqrt{x_{-}(s)^{2}+y_{-}(s)^{2}} \leq\left(l_{3}+l_{4-2}\right) \\
& \left(l_{3}-l_{4-2}\right) \leq \sqrt{x_{+}(s)^{2}+y_{+}(s)^{2}} \leq\left(l_{3}+l_{4-2}\right)
\end{aligned}
$$

where the subscripts in $x_{-}(s)$ and $y_{-}(s)$ and in $x_{+}(s)$ and $y_{+}(s)$ denote the minimum and maximum perturbation about the nominal path, respectively.

Also, the mechanism should be well conditioned even in the perturbed path, therefore

$$
\begin{aligned}
& (\pi / 2)-e_{4} \leq q_{2+}-q_{1+} \leq(\pi / 2)+e_{4} \\
& (\pi / 2)-e_{5} \leq q_{2-}-q_{1-} \leq(\pi / 2)+e_{5}
\end{aligned}
$$

where the subscripts in $q_{i,+}$ and $q_{i,-}, i=1,2$, denote the values of the angles at the maximum and minimum deviated locations, respectively. 
TABLE I

STARTING GUESS, LOWER BOUNDS, AND UPPER BOUNDS FOR THE DESIGN PARAMETERS

\begin{tabular}{cccc}
\hline \hline Parameter & $\begin{array}{c}\text { Starting } \\
\text { guess }\end{array}$ & $\begin{array}{c}\text { Lower } \\
\text { bounds }\end{array}$ & $\begin{array}{c}\text { Upper } \\
\text { bounds }\end{array}$ \\
\hline Length of link 2, $l_{2}$ & $0.05 \mathrm{~m}$ & $0.001 \mathrm{~m}$ & $0.3 \mathrm{~m}$ \\
Length of link 3, $l_{3}$ & $0.05 \mathrm{~m}$ & $0.001 \mathrm{~m}$ & $0.3 \mathrm{~m}$ \\
Length of link 4, $l_{4}$ & $0.05 \mathrm{~m}$ & $0.001 \mathrm{~m}$ & $0.3 \mathrm{~m}$ \\
X coordinate of point C, $C_{x}$ & $-0.05 \mathrm{~m}$ & $-0.5 \mathrm{~m}$ & $0.5 \mathrm{~m}$ \\
Y coordinate of point C, $C_{y}$ & $-0.05 \mathrm{~m}$ & $-0.5 \mathrm{~m}$ & $0.5 \mathrm{~m}$ \\
Balancing weight of link 2, $m_{b 2}$ & $0.1 \mathrm{Kg}$ & $0.0 \mathrm{Kg}$ & $0.5 \mathrm{Kg}$ \\
Balancing weight of link 3, $m_{b 3}$ & $0.1 \mathrm{Kg}$ & $0.0 \mathrm{Kg}$ & $0.5 \mathrm{Kg}$ \\
Extension for balancing of link 2, $l_{b 2,}$ & $0.05 \mathrm{~m}$ & $-0.3 \mathrm{~m}$ & $0.3 \mathrm{~m}$ \\
Extension for balancing of link 3, $l_{t, 3}$ & $0.05 \mathrm{~m}$ & $-0.3 \mathrm{~m}$ & $0.3 \mathrm{~m}$ \\
Motor transmission ratio, $n$ & 5.0 & 1.0 & 10.0 \\
\hline \hline
\end{tabular}

Finally, perturbed path constraints similar to (12) yield

$$
\begin{aligned}
& 60^{\circ} \leq q_{2, C+} \leq 300^{\circ} \\
& 60^{\circ} \leq q_{2, C-} \leq 300^{\circ} .
\end{aligned}
$$

Equations (8)-(16) form the set of optimization inequality constraints for the objective function $f$.

2) Equality Constraints: The objective function (6) contains the gravity vector as seen from the user side, $\tilde{\boldsymbol{G}}=\mathbf{J}^{-T} \boldsymbol{G}$. As discussed previously, $\boldsymbol{G}$ and therefore $\tilde{\boldsymbol{G}}$ can be eliminated by adding two balancing weights on the extensions of links 2 and 3 (see Fig. 3). Elimination of $\boldsymbol{G}$ results in the following optimization equality constraints, functions of the design parameters $m_{\mathrm{b} 2}, m_{\mathrm{b} 3}$ and $l_{\mathrm{b} 2}, l_{\mathrm{b} 3}$

$$
m_{\mathrm{b} 2} l_{\mathrm{b} 2}=m_{4} l_{\mathrm{c} 4}+\sum_{i=5}^{7} m_{i} l_{4-2}-m_{1} l_{2}-m_{\mathrm{c} 2} l_{\mathrm{cb} 2}-m_{2} l_{\mathrm{c} 2}
$$

$$
m_{\mathrm{b} 3} l_{\mathrm{b} 3}=m_{3} l_{\mathrm{c} 3}+\sum_{i=4}^{7} m_{i} l_{3}-m_{\mathrm{c} 3} l_{\mathrm{cb} 3}+m_{1} l_{\mathrm{c} 1}
$$

where $l_{\mathrm{ci}}$ is the $i$ th link mass center location, $m_{\mathrm{ci}}$ is the $i$ th link extension mass, and $l_{\mathrm{cbi}}$ is the $i$ th link extension mass center location.

\section{OPTIMIZATION RESULTS AND DISCUSSION}

To solve the optimization problem, the Matlab optimization toolbox function fmincon was employed. This function is based on a sequential quadratic programming (SQP) method with a number of modifications [15]. In more detail, fmincon finds a constrained minimum of a function of several variables and requires a starting guess, as well as bounds for the design variables. The starting guess, the lower and upper bounds used, and optimization results are displayed in Tables I and II.

For the resulting mechanism, all constraints, (8)-(18), are satisfied. The mechanism follows the nominal and perturbed paths with small condition numbers. Fig. 5 displays the resulting mechanism, path location, and initial and final device configu-
TABLE II

COMPARISON OF RESULTS OF OPTIMIZATION PROCEDURES

\begin{tabular}{cccc}
\hline \hline Parameter & $\begin{array}{c}\text { IOD } \\
\text { results }\end{array}$ & $\begin{array}{c}\text { JSO } \\
\text { results }\end{array}$ & $\begin{array}{c}\text { USOD } \\
\text { results }\end{array}$ \\
\hline Length of link 2, $l_{2}$ & $0.075 \mathrm{~m}$ & $0.060 \mathrm{~m}$ & $0.023 \mathrm{~m}$ \\
Length of link 3, $l_{3}$ & $0.135 \mathrm{~m}$ & $0.110 \mathrm{~m}$ & $0.093 \mathrm{~m}$ \\
Length of link 4, $l_{4}$ & $0.230 \mathrm{~m}$ & $0.170 \mathrm{~m}$ & $0.103 \mathrm{~m}$ \\
X coordinate of point $\mathrm{C}, C_{r}$ & - & $0.064 \mathrm{~m}$ & $0.053 \mathrm{~m}$ \\
Y coordinate of point $\mathrm{C}, C_{y}$ & - & $-0.126 \mathrm{~m}$ & $-0.124 \mathrm{~m}$ \\
Balancing weight of link 2, $m_{b 2}$ & - & - & $0.080 \mathrm{Kg}$ \\
Balancing weight of link 3, $m_{b: 3}$ & - & - & $0.241 \mathrm{Kg}$ \\
Extension for balancing of link $2, l_{b: 2}$ & - & - & $0.039 \mathrm{~m}$ \\
Extension for balancing of link 3, $l_{t, 3}$ & - & - & $0.052 \mathrm{~m}$ \\
Motor transmission ratio, $n$ & - & - & 4.750 \\
\hline \hline
\end{tabular}

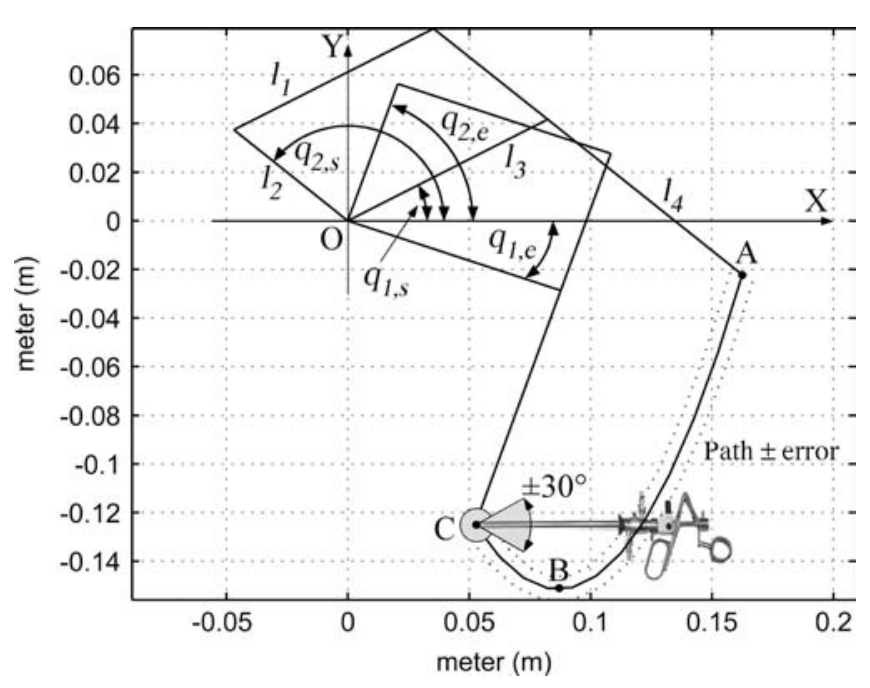

Fig. 5. Optimization results showing the initial and final device configuration.

ration. In this figure, subscripts $s$ and $e$ in $q_{i, s}$ and $q_{i, e}, i=1,2$, denote the values of the angles at the start and the end of the nominal path $\mathrm{ABC}$ ending at the optimum location.

Table II also displays the optimization results of the IOD, and JSO optimizations, described earlier. An empty cell indicates that the corresponding parameter was not optimized.

To compare the performance of the mechanisms corresponding to the various optimization procedures, the condition number and the norm of the parasitic forces/torques along the nominal path $\mathrm{ABC}$ are calculated and given as function of the $k$ th path segment and presented in Fig. 6.

It is clear that USOD gives the best results. The norm of the parasitic forces/torques along the path $\mathrm{ABC}$ is reduced by $20 \%$ relative to the JSO and by $60 \%$ relative to the IOD, see Fig. 6(b). The condition number is reduced by $10 \%-15 \%$ relative to the JSO, see Fig. 6(a).

Fig. 6(a) also shows that the condition number in IOD presents a large difference between the path starting point $A$ and the path ending point $C$. This is expected because in IOD the nominal path was fixed in the device's workspace and only the condition number at point $C$ was optimized. 


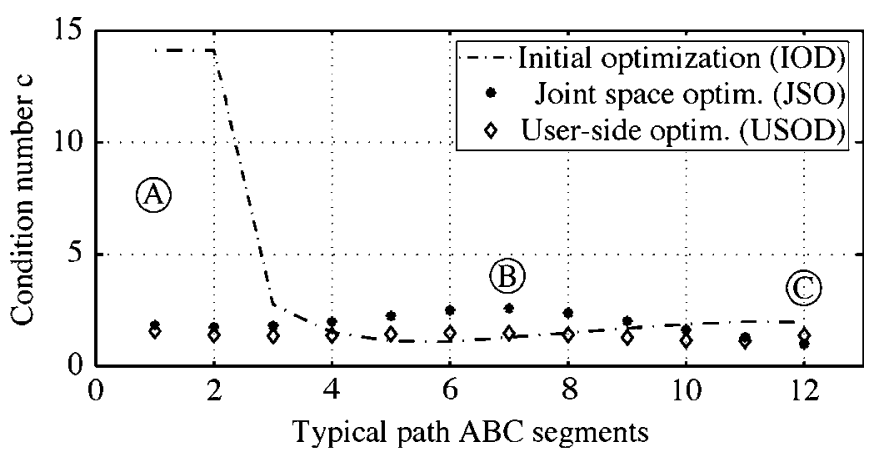

(a)

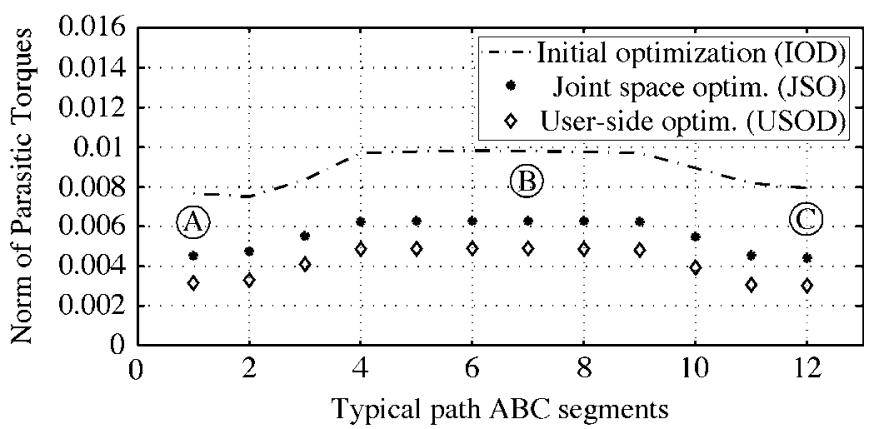

(b)

Fig. 6. Optimization results evaluation. USOD results are the best. (a) Condition number c. (b) Norm of parasitic torques.

In USOD, the condition number at path point $C$, where the main operation occurs, is very small and close to the ideal 1 , i.e., $c=1.12$. At this point, $q_{2}-q_{1}=78.5^{\circ}$ and $q_{2}=70^{\circ}$, both within the constraints. The peak in Fig. 6 occurs on path point $B$, where the mechanism is stretched out to follow the path, see Fig. 5. This occurs due to the weighting factors used in the objective function (6) and reflects the fact that point $B$ is not as important as $C$, being only a via path point of the insertion phase.

An important performance criterion in haptic interfaces is the apparent mass of the device, i.e., the mass felt by a user when the device is accelerated. It was calculated that the apparent mass in the $X$ - and $Y$-axes is about 255 and $110 \mathrm{~g}$, respectively, for IOD, 163 and $84 \mathrm{~g}$ for JSO, i.e., a reduction of about $36 \%$, and finally, 142 and $72 \mathrm{~g}$ for USOD, which corresponds to a further reduction of about $15 \%$. Therefore, the employed methodology resulted in significant improvements in the performance of the device.

In most optimizations, a question that needs to be addressed is whether the results correspond to the true optimum. This holds here too, since the SQP method yields only local optima. To find the true minimum, the optimization procedure is performed several times using different bounding ranges for the design parameters. The best result of all optimization runs is taken to be the global optimum.

To verify that a global minimum was computed, all design parameters, but one each time, were kept at the assumed optimum, while the objective function was computed from the lower to the upper bound of the remaining parameter. Fig. 7 displays the results of these computations for the first five de-

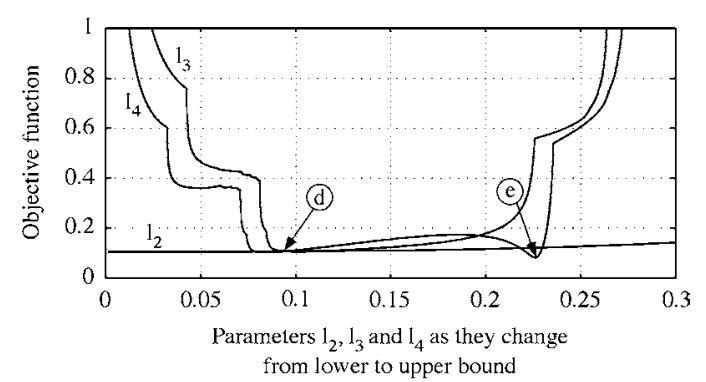

(a)

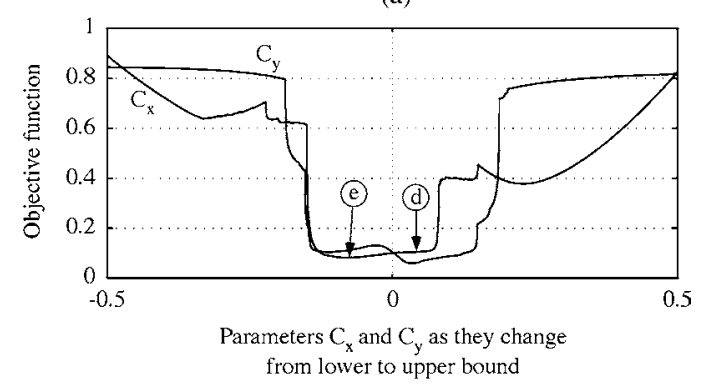

(b)

Fig. 7. Objective function, as some of the design parameters change from lower to upper bound. (a) Parameters $l_{2}, l_{3}$, and $l_{4}$. (b) Parameters $C_{x}$ and $C_{y}$.

sign parameters $\left(l_{2}, l_{3}, l_{4}, C_{x}, C_{y}\right)$. The first observation is that the calculated minimum of the objective function appears to be smaller than that of the optimization results in Table II. However, this minimum is not acceptable because of the violation of some kinematical or implementation constraint. For example, the optimization procedure yields a minimum for the objective function when parameter value $C_{x}=0.053 \mathrm{~m}$ [point $d$ in Fig. 7(b)]. In the same figure, a lower value for the objective function occurs when $C_{x}=-0.079 \mathrm{~m}$, [point $e$ in Fig. 7(b)]. However, this calculated minimum does not satisfy constraint (12). This is also the case for $l_{3}$ in Fig. 7(a), etc.

\section{Device IMPLEMENTATION AND Discussion}

The new optimized mechanism was constructed based on the optimization results in Table II and is presented in Fig. 8(a). Fig. 8(b) shows the new and initial mechanisms side by side. It is obvious that the optimized haptic device is significantly smaller compared to the initial one, having link lengths about $20 \%$ shorter, yet it delivers an improved performance.

In addition to the geometrical and mass design parameters, the procedure yields an optimal transmission ratio $n$ between the five-bar motors and links 2 and 3. This ratio, along with predetermined force/torque requirements, see [6], also yields the necessary motor torques, and, according to a predefined table of candidate motors, the suitable motors.

In more detail, it was found by experiments, see [6], that the upper limit of the forces in $X$ - and $Y$-axes is $4.5 \mathrm{~N}$. Given that the joint speeds are very low, one can choose motors based on the starting (stall) torque only. This leads to the selection of a $60-\mathrm{W}$ Maxon dc motor with a torque constant of $53.8 \mathrm{mN} \cdot \mathrm{m} / \mathrm{A}$, maximum continuous torque of $96.6 \mathrm{mN} \cdot \mathrm{m}$, a nominal voltage of $48 \mathrm{~V}$, and rotor inertia of $32.2 \mathrm{~g} \cdot \mathrm{cm}^{2}$. The motors are equipped with a digital MR encoder with 1024 counts per turn. 


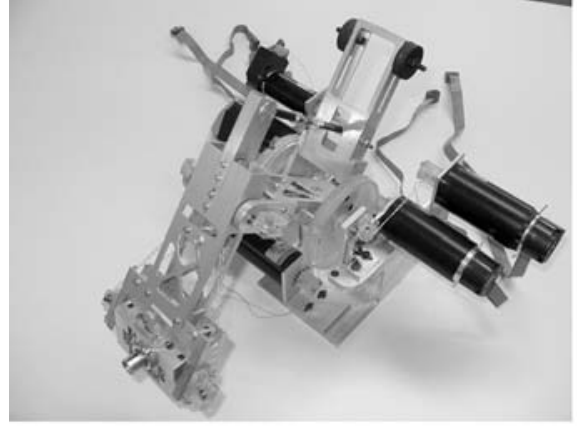

(a)

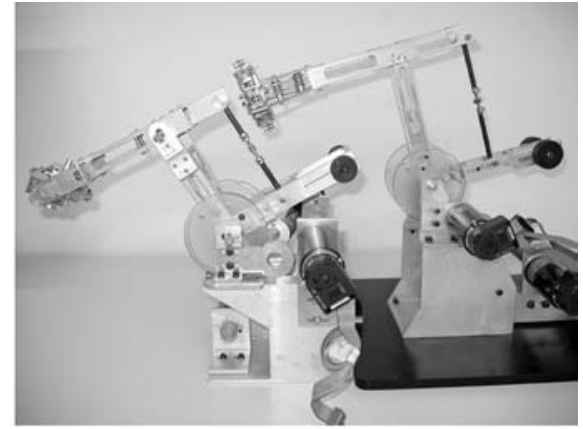

(b)

Fig. 8. (a) Assembled optimized device and (b) new and initial mechanism side by side.

\section{CONCLUSION}

A design methodology aiming at the maximization of transparency in haptic devices, as seen from the user side, was developed. The focus of the method is on endpoint side fidelity and optimizes not only mechanism dimensions, but also relevant design parameters including relative position of endpoint desired path to device location, motor transmission ratios, and rotor inertias and motor sizes. The methodology was exemplified using a 5-DOF haptic device, part of a training medical urological simulator, and is applicable to any haptic mechanism. A multivariable optimization approach was employed. The objective function was based on the device dynamics and motor and transmission parameters, as seen from the user side. The kinematical and operational constraints were described and discussed. Using the optimization results, a new 5-DOF haptic device was constructed and was shown to be improved significantly with respect to one designed with a standard approach.

\section{REFERENCES}

[1] M. P. Laguna, M. Hatzinger, and J. Rassweiler, "Simulators and endourological training," Curr. Opin. Urol., vol. 12, pp. 209-215, 2002.

[2] T. Massie and J. K. Salisbury, "The phantom haptic interface: A device for probing virtual objects," in Proc. ASME Winter Annu. Meeting, Symp. Haptic Interfaces Virtual Environ. Teleoperator Syst., 1994, pp. 295-301.

[3] V. Hayward et al., Freedom-7: A high fidelity seven axis haptic device with application to surgical training, in Experimental Robot. V, A. Casals and A. T. de Almeida, Eds. Springer-Verlag, Berlin, Germany, 1998, pp. $445-456$.

[4] R. Baumann et al., "The PantoScope: A spherical remote-center-ofmotion parallel manipulator for force reflection," in Proc. IEEE ICRA, vol. 1, 1997, pp. 718-723.

[5] U. Kühnapfel et al., The Karlsruhe endoscopic surgery trainer as an example for virtual reality in medical education,Minimally Invasive Therapy and Allied Technologies. Oxford, U.K.: Blackwell, 1997, pp. 122125 .

[6] K. Vlachos, E. Papadopoulos, and D. N. Mitropoulos, "Design and implementation of a haptic device for training in urological operations," IEEE Trans. Robot. Autom., vol. 19, no. 5, pp. 801-809, Oct. 2003.

[7] O. Khatib and A. Bowling, "Optimization of the inertial and acceleration characteristics of manipulators," in Proc. IEEE ICRA, vol. 4, 1996, pp. 2883-2889.

[8] P. Millman and E. Colgate, "Design of a four degree-of-freedom forcereflecting manipulandum with a specified force/torque workspace," in Proc. IEEE ICRA, vol. 2, 1991, pp. 1488-1493.

[9] L. Stocco, S. E. Salcudean, and F. Sassani, "Fast constrained global optimization of robot parameters," Robotica, vol. 16, pp. 595-605, 1999.
[10] L. J. Stocco, S. E. Salcudean, and F. Sassani, "Optimal kinematic design of a haptic pen," IEEE/ASME Trans. Mechatronics, vol. 6, no. 3, pp. 210 220, Sep. 2001.

[11] V. Hayward, J. Choksi, G. Lanvin, and C. Ramstein, Design and multiobjective optimization of a linkage for a haptic interface in Advances in Robot Kinematics and Comput. Geometry, A. J. Lenarcic and B. B. Ravani, Eds. Norwell, MA: Kluwer, 1994, pp. 359-368.

[12] R. Kurtz and V. Hayward, "Multiple-goal kinematic optimization of a parallel spherical mechanism with actuator redundancy," IEEE Trans. Robot. Autom., vol. 8, no. 5, pp. 644-651, Oct. 1992.

[13] M. Ueberle, N. Mock, and M. Buss, "ViSHaRD10, a novel hyperredundant haptic interface," in Proc. 12th Int. Symp. Haptic Interfaces Virtual Environ. Teleoperator Syst., 2004, pp. 58-65.

[14] K. Vlachos, E. Papadopoulos, and D. N. Mitropoulos, "Mass/inertia and joint friction minimization for a low-force five-DOF haptic device," in Proc. IEEE ICRA, vol. 1, 2004, pp. 286-291.

[15] P. E. Gill, W. Murray, and M. H. Wright, Practical Optimization. London, U.K.: Academic, 1981.

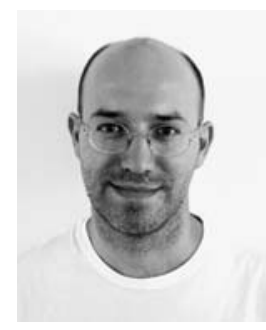

Kostas Vlachos received the B.Sc. degree in electrical engineering from the Technical University of Dresden, Dresden, Germany, in 1993 and the M.S. and $\mathrm{Ph} . \mathrm{D}$. degrees in mechanical engineering from the National Technical University of Athens (NTUA), Athens, Greece, in 2000 and 2004, respectively.

From 1996 to 1998, he worked as a Software Analyst. Currently, he is a Postdoctoral Researcher with the Mechanical Engineering Department, NTUA. His research interests include haptic mechanisms, medical simulation, and control of robotic mechanisms.

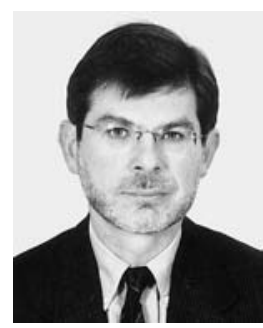

Evangelos Papadopoulos (''83-M'91-SM'97) received the diploma from the National Technical University of Athens (NTUA), Athens, Greece, in 1981 and the M.S. and Ph.D. degrees from the Massachusetts Institute of Technology, Cambridge, in 1983 and 1991, respectively, all in mechanical engineering.

From 1985 to 1987, he was an Analyst with the Hellenic Navy, Athens, Greece. In 1991, he joined McGill University and the Center for Intelligent Machines (CIM) as an Assistant Professor and received tenure in 1997. Currently, he is an Associate Professor with the Mechanical Engineering Department, NTUA. He has published more than 120 technical articles in journals and refereed conference proceedings. His research interests include robotics, modeling and control of dynamic systems, mechatronics, and design.

Dr. Papadopoulos is a Senior member of the AIAA and a member of the ASME. He serves as an Associate Editor for the IEEE TRANSACTIONS ON RовотіCS and the Machine and Mechanism Theory. He has served as a Guest Editor to the IEEE/ASME TRANSACTIONS ON MECHATRONICS. He is a member of the Technical Chamber of Greece (TEE) and the Sigma Xi. 